\title{
Space astrometry with the Milli-Arcsecond Pathfinder Survey: mission overview and science possibilities
}

\author{
Ralph A. Gaume ${ }^{1}$, Bryan Dorland ${ }^{1}$, Valeri V. Makarov ${ }^{2}$, Norbert \\ Zacharias $^{1}$, Kenneth J. Johnston ${ }^{1}$, and Gregory S. Hennessy ${ }^{1}$ \\ ${ }^{1}$ Astrometry Depertment, US Naval Observatory (USNO), \\ 3450 Massachusetts Avenue NW, Washington DC 20392-5420, USA \\ email: rgaume,bdorland,nz,kjj,gsh@usno.navy.mil \\ ${ }^{2}$ Michelson Science Center, California Institute of Technology, \\ MS 301 486, 4800 Oak Grove Drive, Pasadena, CA 91109, USA \\ email: valeri.v.makarov-119624@jpl.nasa.gov
}

\begin{abstract}
We present a mission overview and science possibilities of space astrometry with the Milli-Arcsecond Pathfinder Survey (MAPS).
\end{abstract}

Keywords. astrometry, surveys, reference systems, stars: kinematics, instrumentation

The Milli-Arcsecond Pathfinder Survey (MAPS) mission is a space-based, all-sky astrometric and photometric survey from $2^{\text {nd }}$ through $15^{\text {th }}$ magnitude with a 2010 launch date goal. The primary mission goal for MAPS is the generation of a 1 mas all-sky astrometric catalog for the 2010 epoch.

The instrument consists of a $15 \mathrm{~cm}$ telescope and large (64 megapixel) active pixel sensor focal plane with associated processing electronics carried aboard a microsatellite bus in a $900 \mathrm{~km}$ sun-synchronous low Earth orbit.

$M A P S$ technology, including the very large format detector, the onboard processing electronics, and next generation space-based GPS-receiver, will serve as a pathfinder in support of future space missions.

A 1 mas (or better) all-sky survey through $15^{\text {th }}$ magnitude will have a tremendous impact on our current understanding of the galaxy and stellar astrophysics. MAPS science topics include:

(i) a kinematic and photometric exploration of the nearest star forming regions and associations; an understanding of the dynamics and membership of nearby open clusters;

(ii) a survey of nearby stars that addresses the 130 missing systems within $10 \mathrm{pc}$;

(iii) recalibration of the cosmic distance scale via distances to nearby clusters, and the period-luminosity relationship using high accuracy proper motion (Hipparcos and MAPS positions and a twenty year baseline) and parallax measurements;

(iv) discovery of giant planets and brown dwarfs orbiting nearby stars; kinematic detection of galactic cannibalism and mergers in the Milky Way; and

$(v)$ discovery of low-mass black holes and neutron stars in astrometric binaries. 